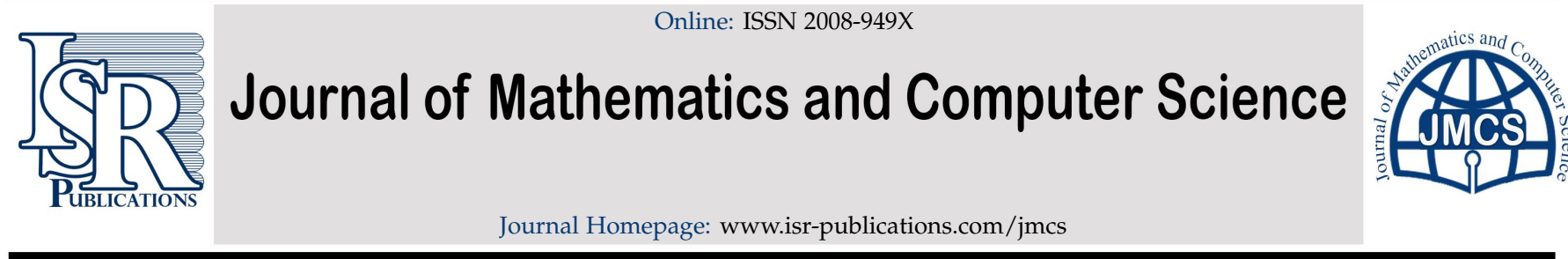

\title{
Dynamic behaviors of a two-species competitive system with nonlinear inter-inhibition terms and infinite delay
}

Runxin $\mathrm{Wu}^{\mathrm{a}, *}$, Zhiqing $\mathrm{Gao}^{\mathrm{b}}$, Fengde Chen ${ }^{\mathrm{b}}$

${ }^{a}$ Mathematics and Physics Institute, Fujian University of Technology, Fuzhou, Fujian, 350014, P. R. China.

${ }^{b}$ College of Mathematics and Computer Science, Fuzhou University, Fuzhou, Fujian, 350116, P. R. China.

\begin{abstract}
Sufficient conditions are obtained for the global attractivity of the positive equilibrium and boundary equilibria of the following two-species competitive system with nonlinear inter-inhibition terms

$$
\frac{d y_{1}(t)}{d t}=y_{1}(t)\left[r_{1}-a_{1} y_{1}-\frac{b_{1} \int_{-\infty}^{t} K_{2}(t-s) y_{2}(s) d s}{1+\int_{-\infty}^{t} K_{2}(t-s) y_{2}(s) d s}\right], \quad \frac{d y_{2}(t)}{d t}=y_{2}(t)\left[r_{2}-a_{2} y_{2}-\frac{b_{2} \int_{-\infty}^{t} K_{1}(t-s) y_{1}(s) d s}{1+\int_{-\infty}^{t} K_{1}(t-s) y_{1}(s) d s}\right],
$$

where $r_{i}, a_{i}, b_{i}, i=1,2$ are all positive constants. The results not only improve but also complement the main results of [B. G. Chen, J. Math. Computer Sci., 16 (2016), 481-494].
\end{abstract}

Keywords: Competition, nonlinear inter-inhibition terms, global attractivity, infinite delay.

2020 MSC: 34C25, 92D25, 34D20.

(C)2020 All rights reserved.

\section{Introduction}

The aim of this paper is to investigate the global dynamic behaviors of the following two-species competitive system with nonlinear inter-inhibition terms and infinite delay

$$
\begin{aligned}
& \frac{d y_{1}(t)}{d t}=y_{1}(t)\left[r_{1}-a_{1} y_{1}-\frac{b_{1} \int_{-\infty}^{t} K_{2}(t-s) y_{2}(s) d s}{1+\int_{-\infty}^{t} K_{2}(t-s) y_{2}(s) d s}\right], \\
& \frac{d y_{2}(t)}{d t}=y_{2}(t)\left[r_{2}-a_{2} y_{2}-\frac{b_{2} \int_{-\infty}^{t} K_{1}(t-s) y_{1}(s) d s}{1+\int_{-\infty}^{t} K_{1}(t-s) y_{1}(s) d s}\right],
\end{aligned}
$$

*Corresponding author

Email addresses: runxinwu@163.com (Runxin Wu), 99949995@qq.com (Zhiqing Gao), fdchen@263.net (Fengde Chen)

doi: $10.22436 /$ jmcs.021.01.05

Received: 2020-01-28 Revised: 2020-02-12 Accepted: 2020-02-19 
where $r_{i}, a_{i}, b_{i}, i=1,2$ are all positive constants. The delay kernels $K_{i}:[0,+\infty) \rightarrow(0,+\infty), i=1,2$ are continuous functions such that

$$
\int_{0}^{+\infty} K_{i}(s) d s=1
$$

We shall consider (1.1) together with the initial conditions

$$
y_{i}(s)=\phi_{i}(s), s \in(-\infty, 0], i=1,2,
$$

where $\phi_{i} \in \mathrm{BC}^{+}, i=1,2$ and

$$
\mathrm{BC}^{+}=\{\phi \in \mathrm{C}((-\infty, 0],[0,+\infty)): \phi(0)>0 \text { and } \phi \text { isbounded }\} .
$$

It is well known that by the fundamental theory of functional differential equations [12], system (1.1) has a unique solution $\left(y_{1}(t), y_{2}(t)\right)$ satisfying the initial condition (1.2).

Traditional two species Lotka-Volterra competition model takes the form

$$
\frac{d y_{1}(t)}{d t}=y_{1}(t)\left[r_{1}-a_{1} y_{1}-b_{1} y_{2}\right], \quad \frac{d y_{2}(t)}{d t}=y_{2}(t)\left[r_{2}-a_{2} y_{2}-b_{2} y_{1}\right]
$$

The system may exhibits the coexists, partial survival or bistable dynamic behaviors, see [20]. Based on this model, many scholars ([1,3-34]) proposed more complicated competition model, and investigated the dynamic behaviors of the system.

Stimulated by the functional response of the predator prey system, Gopalsamy [11] proposed the following two species competitive system with nonlinear inter-inhibition terms

$$
\frac{d y_{1}(t)}{d t}=y_{1}(t)\left[r_{1}-a_{1} y_{1}-\frac{b_{1} y_{2}}{1+y_{2}}\right], \quad \frac{d y_{2}(t)}{d t}=y_{2}(t)\left[r_{2}-a_{2} y_{2}-\frac{b_{2} y_{1}}{1+y_{1}}\right],
$$

where $r_{i}, a_{i}, b_{i}, i=1,2$ are all positive constants. Recently, Wang et al. [24] studied the dynamic behaviors of the nonautonomous case of system (1.3), i.e, the following modelling

$$
\frac{d y_{1}(t)}{d t}=y_{1}(t)\left[r_{1}(t)-a_{1}(t) y_{1}-\frac{b_{1}(t) y_{2}}{1+y_{2}}\right], \quad \frac{d y_{2}(t)}{d t}=y_{2}(t)\left[r_{2}(t)-a_{2}(t) y_{2}-\frac{b_{2}(t) y_{1}}{1+y_{1}}\right]
$$

where $y_{i}(i=1,2)$ are the population densities of two competing species at time $t ; r_{i}(t)(i=1,2)$ are the intrinsic growth rates of species $i ; a_{i}(i=1,2)$ are the rates of intraspecific competition of the first and second species, respectively; $b_{\mathfrak{i}}(t)(i=1,2)$ are the rates of interspecific competition of the first and second species, respectively. For the almost periodic case, the authors investigated the existence and global asymptotic stability of positive almost periodic solutions of the system (1.4). However, in their prove of the stability, the authors had investigated the stability property of the system (1.4) by constructing some suitable Lyapunov function, this, as was pointed out by Baoguo Chen[2], generally speaking, may lead to the the conditions become very complicated, the additional condition, to some extent, is necessary. In [2], by using the iterative method and the theory of differential inequality, Baoguo Chen obtained sufficient conditions which ensure the global attractivity of the positive equilibrium and boundary equilibrium of the system (1.3), respectively. Also, at the end of the paper, he pointed out: "We mention here that a suitable population model should incorporate some past state of the species, and this will lead to a system with delay. Whether the delay has positive or negative influence on the dynamic behaviors of the system is still unknown, we leave this for future investigation." However, to the best of the authors knowledge, to this day, still no scholars propose the competition system with nonlinear inter-inhibition terms and infinite delay, this motivate us to propose the system (1.1).

The aim of this paper is, by further developing the analysis technique of $[1-3,5,6,8,9]$ and using the differential inequality theory, to investigate the global dynamic behaviors of the system (1.1). More precisely, we will prove the following results. 
Theorem 1.1. Assume that the following inequalities

$$
r_{1}\left(a_{2}+r_{2}\right)>b_{1} r_{2}, r_{2}\left(a_{1}+r_{1}\right)>b_{2} r_{1}
$$

hold, then system (1.1) admits a unique positive equilibrium $\left(y_{1}^{*}, y_{2}^{*}\right)$, which is globally attractive, that is, for any positive solution $\left(\mathrm{y}_{1}(\mathrm{t}), \mathrm{y}_{2}(\mathrm{t})\right)$ of system (1.1) with the initial condition (1.2), one has:

$$
\lim _{t \rightarrow+\infty} y_{i}(t)=y_{i}^{*}, i=1,2 \text {. }
$$

Remark 1.2. In addition to (1.5), Baoguo Chen [2] further assume that one of the following conditions holds,

$$
\begin{aligned}
& a_{2}-b_{2}+r_{2} \neq 0 ; \\
& a_{2}-b_{2}+r_{2}=0, a_{1} r_{2}-a_{2} r_{1}>0,
\end{aligned}
$$

then system (1.4) admit a unique positive equilibrium $\left(y_{1}^{*}, y_{2}^{*}\right)$, which is globally attractive. Obviously, our result not only generalize the main result of Baoguo Chen [2] to the infinite delay case, but also supplement and complement the main result of Baoguo Chen, by dropping off the unnecessary condition.

As a direct corollary of Theorem 1.1, we have following.

Corollary 1.3. Assume that the following inequalities

$$
r_{1}>b_{1}, r_{2}>b_{2}
$$

hold, then system (1.1) admit a unique positive equilibrium $\left(\mathrm{y}_{1}^{*}, \mathrm{y}_{2}^{*}\right)$, which is globally attractive, that is, for any positive solution $\left(\mathrm{y}_{1}(\mathrm{t}), \mathrm{y}_{2}(\mathrm{t})\right)$ of system (1.1) with the initial condition (1.5), one has:

$$
\lim _{t \rightarrow+\infty} y_{i}(t)=y_{i}^{*}, i=1,2 \text {. }
$$

Theorem 1.4. Assume that the following inequalities

$$
r_{1}\left(a_{2}+r_{2}\right)>b_{1} r_{2}, r_{2}-\frac{b_{2} m_{1}}{1+m_{1}}<0
$$

hold, where

$$
\mathrm{m}_{1}=\frac{\mathrm{r}_{1}-\frac{\mathrm{b}_{1} \mathrm{M}_{2}}{1+\mathrm{M}_{2}}}{\mathrm{a}_{1}}, \quad \mathrm{M}_{2}=\frac{\mathrm{r}_{2}}{\mathrm{a}_{2}}
$$

then

$$
\lim _{t \rightarrow+\infty} y_{1}(t)=\frac{r_{1}}{a_{1}}, \lim _{t \rightarrow+\infty} y_{2}(t)=0 .
$$

Theorem 1.5. Assume that the following inequalities

$$
r_{1}-\frac{b_{1} m_{2}}{1+m_{2}}<0, r_{2}\left(a_{1}+r_{1}\right)>b_{2} r_{1}
$$

hold, where

$$
\mathrm{m}_{2}=\frac{\mathrm{r}_{2}-\frac{\mathrm{b}_{2} \mathrm{M}_{1}}{1+\mathrm{M}_{1}}}{\mathrm{a}_{2}}, \mathrm{M}_{1}=\frac{\mathrm{r}_{1}}{\mathrm{a}_{1}}
$$

then

$$
\lim _{t \rightarrow+\infty} y_{1}(t)=0, \lim _{t \rightarrow+\infty} y_{2}(t)=\frac{r_{2}}{a_{2}} .
$$

Remark 1.6. Theorems 1.4 and 1.5 are the same as that of the conclusions of Baoguo Chen [2]. Compared with the corresponding results of Baoguo Chen [2], we can draw the conclusion: In system (1.1), delay has no influence to the dynamic behaviors of the system. Delay is profitless and harmless to the persistent or extinction of the species.

The rest rest of the paper is arranged as follows. We will introduce some useful Lemmas in the next section, and then prove the main results in Section 3. 


\section{Lemmas}

Now let's state several lemmas which will be useful in the proving of main results.

Lemma 2.1. Assume that (1.5) holds, then system (1.1) admits a unique positive equilibrium $\left(y_{1}^{*}, y_{2}^{*}\right)$.

Proof. Chen [2] had showed that under the assumption (1.6) and (1.7) or (1.8) hold, then system (1.1) admits a unique positive equilibrium $\left(y_{1}^{*}, y_{2}^{*}\right)$.

We only mention here that one of the condition (1.7) or (1.8) always holds. Indeed, if (1.7) holds, then the conclusion holds. Assume that (1.7) do not hold, then $a_{2}-b_{2}+r_{2}=0$, thus, by using the second inequality in (1.5), we have

$$
a_{1} r_{2}-a_{2} r_{1}=a_{1} r_{2}-\left(b_{2}-r_{2}\right) r_{1}=r_{2}\left(a_{1}+r_{1}\right)-b_{2} r_{1}>0,
$$

that is, (1.8) holds. This ends the proof of Lemma 2.1.

As a direct corollary of Lemma 2.2 of Chen cite13, we have the following lemma.

Lemma 2.2. If $\mathrm{a}>0, \mathrm{~b}>0$ and $\dot{x} \geqslant x(\mathrm{~b}-\mathrm{ax})$, when $\mathrm{t} \geqslant 0$ and $x(0)>0$, we have

$$
\liminf _{t \rightarrow+\infty} x(t) \geqslant \frac{b}{a} \text {. }
$$

If $\mathrm{a}>0, \mathrm{~b}>0$ and $\dot{\mathrm{x}} \leqslant x(\mathrm{~b}-\mathrm{ax})$, when $\mathrm{t} \geqslant 0$ and $x(0)>0$, we have

$$
\limsup _{t \rightarrow+\infty} x(t) \leqslant \frac{b}{a}
$$

Following Lemma 2.3 is Lemma 3 of Francisco Montes de Oca and Miguel Vivas [10].

Lemma 2.3. Let $x: R \rightarrow R$ be a bounded nonnegative continuous function, and let $\mathrm{k}:[0,+\infty) \rightarrow(0,+\infty)$ be a continuous kernel such that $\int_{0}^{\infty} k(s) d s=1$. Then

$$
\liminf _{t \rightarrow+\infty} x(t) \leqslant \liminf _{t \rightarrow+\infty} \int_{-\infty}^{t} k(t-s) x(s) d s \leqslant \limsup _{t \rightarrow+\infty} \int_{-\infty}^{t} k(t-s) x(s) d s \leqslant \limsup _{t \rightarrow+\infty} x(t) .
$$

Lemma 2.4. Let $\left(y_{1}(t), y_{2}(t)\right)$ be any solution of (1.1)-(1.2), then $y_{i}(t)>0$ for all $i=1,2$ in maximal interval of existence of the solution.

Proof. We note that from (1.1), one has

$$
\frac{d y_{1}(t)}{d t}=P_{1}(t) y_{1}(t), \quad \frac{d y_{2}(t)}{d t}=P_{2}(t) y_{2}(t)
$$

where

$$
P_{1}(t)=r_{1}-a_{1} y_{1}-\frac{b_{1} \int_{-\infty}^{t} K_{2}(t-s) y_{2}(s) d s}{1+\int_{-\infty}^{t} K_{2}(t-s) y_{2}(s) d s}, \quad P_{2}(t)=r_{2}-a_{2} y_{2}-\frac{b_{2} \int_{-\infty}^{t} K_{1}(t-s) y_{1}(s) d s}{1+\int_{-\infty}^{t} K_{1}(t-s) y_{1}(s) d s} .
$$

Hence,

$$
y_{1}(t)=y_{1}(0) \exp \left\{\int_{0}^{t} P_{1}(s) d s\right\}>0, \quad y_{2}(t)=y_{2}(0) \exp \left\{\int_{0}^{t} P_{2}(s) d s\right\}>0
$$

This ends the proof of Lemma 2.4 .

Lemma 2.4 shows that $x_{i}(t)>0, u_{i}(t)>0$ for all $i=1,2$ in maximal interval of existence of the solution. For the rest of the paper, the solution of system (1.1) satisfying the initial conditions (1.2) is said to be positive. 


\section{Proof of the main results}

Now we are in the position of proving the main results of this paper.

Proof of Theorem 1.1. It follows from (1.5) that there exists a $\varepsilon>0$ enough small such that

$$
r_{1}>\frac{b_{1}\left(\frac{r_{2}}{a_{2}}+\varepsilon\right)}{1+\left(\frac{r_{2}}{a_{2}}+\varepsilon\right)}+a_{1} \varepsilon, \quad r_{2}>\frac{b_{2}\left(\frac{r_{1}}{a_{1}}+\varepsilon\right)}{1+\left(\frac{r_{1}}{a_{1}}+\varepsilon\right)}+a_{2} \varepsilon .
$$

Let $\left(y_{1}(t), y_{2}(t)\right)$ be any positive solution of system (1.1) with initial condition (1.5). From system (1.1) it follows that

$$
\frac{d y_{i}(t)}{d t} \leqslant y_{i}(t)\left[r_{i}-a_{i} y_{i}\right]
$$

Thus, as a direct corollary of Lemma 2.2, according to (3.1), one has

$$
\limsup _{t \rightarrow+\infty} y_{i}(t) \leqslant \frac{r_{i}}{a_{i}} .
$$

It follows from Lemma 2.3 that

$$
\limsup _{t \rightarrow+\infty} \int_{-\infty}^{t} K_{i}(t-s) y_{i}(s) d s \leqslant \limsup _{t \rightarrow+\infty} y_{i}(t) \leqslant \frac{r_{i}}{a_{i}} .
$$

Hence, for enough small $\varepsilon>0$, which satisfies (3.1), it follows from (3.3) and (3.4) that there exists a $T_{1}>0$ such that for all $t \geqslant T_{1}$

$$
\begin{array}{r}
y_{i}(t)<\frac{r_{i}}{a_{i}}+\varepsilon \stackrel{\text { def }}{=} M_{i}^{(1)}, i=1,2, \\
\int_{-\infty}^{t} K_{i}(t-s) y_{i}(s) d s<\frac{r_{i}}{a_{i}}+\varepsilon \stackrel{\text { def }}{=} M_{i}^{(1)}, i=1,2 .
\end{array}
$$

One could easily see that the function $f_{i}(x)=\frac{b_{i} x}{1+x}, i=1,2$ are the increasing function, and so, for $t>T_{1}$, it follows from the first equation of system (1.1) and (3.6) that

$$
\frac{d y_{1}(t)}{d t} \geqslant y_{1}(t)\left[r_{1}-a_{1} y_{1}-\frac{b_{1} M_{2}^{(1)}}{1+M_{2}^{(1)}}\right]
$$

Thus, as a direct corollary of Lemma 2.2, according to (3.7), one has

$$
\liminf _{t \rightarrow+\infty} y_{1}(t) \geqslant \frac{r_{1}-\frac{b_{1} M_{2}^{(1)}}{1+M_{2}^{(1)}}}{a_{1}} .
$$

It follows from (2.1) and (3.8) that

$$
\liminf _{t \rightarrow+\infty} \int_{-\infty}^{t} K_{1}(t-s) y_{1}(s) d s \geqslant \liminf _{t \rightarrow+\infty} y_{1}(t) \geqslant \frac{r_{1}-\frac{b_{1} M_{2}^{(1)}}{1+M_{2}^{(1)}}}{a_{1}}
$$

Hence, for enough small $\varepsilon>0$, which satisfies (3.1), it follows from (3.1) and (3.6) that there exists a $\mathrm{T}_{2}^{\prime}>0$ 
such that for all $t>T_{2}$

$$
\begin{aligned}
& y_{1}(t)> \frac{r_{1}-\frac{b_{1} M_{2}^{(1)}}{1+M_{2}^{(1)}}}{a_{1}}-\varepsilon \stackrel{\text { def }}{=} m_{1}^{(1)} \text { for } t>T_{2}^{\prime}, \\
& \int_{-\infty}^{t} K_{1}(t-s) y_{1}(s) d s>\frac{r_{1}-\frac{b_{1} M_{2}^{(1)}}{1+M_{2}^{(1)}}}{a_{1}}-\varepsilon \stackrel{\text { def }}{=} m_{1}^{(1)} \text { for } t>T_{2}^{\prime} .
\end{aligned}
$$

Similarly, for above $\varepsilon>0$, it follows from the second equation of system (1.1) that there exists a $T_{2}>T_{2}^{\prime}$ such that

$$
\begin{aligned}
y_{2}(t)>\frac{r_{2}-\frac{b_{2} M_{1}^{(1)}}{1+M_{1}^{(1)}}}{a_{2}}-\varepsilon \stackrel{\text { def }}{=} m_{2}^{(1)} \text { for } t>T_{2}, \\
\int_{-\infty}^{t} K_{2}(t-s) y_{2}(s) d s>\frac{r_{2}-\frac{b_{2} M_{1}^{(1)}}{1+M_{1}^{(1)}}}{a_{2}}-\varepsilon \stackrel{\text { def }}{=} m_{2}^{(1)} \text { for } t>T_{2} .
\end{aligned}
$$

For $t>T_{2}$, it follows from the first equation of system (1.1), the strict increasing of function $f_{i}(x)=$ $\frac{b_{i} x}{1+x}, i=1,2$ and (3.9) that

$$
\frac{d y_{1}(t)}{d t} \leqslant y_{1}(t)\left[r_{1}-a_{1} y_{1}-\frac{b_{1} m_{2}^{(1)}}{1+m_{2}^{(1)}}\right] \text {. }
$$

Thus, as a direct corollary of Lemma 2.2, according to (3.10), one has

$$
\limsup _{t \rightarrow+\infty} y_{1}(t) \leqslant \frac{r_{1}-\frac{b_{1} m_{2}^{(1)}}{1+m_{2}^{(1)}}}{a_{1}}
$$

It follows from Lemma 2.3 that

$$
\limsup _{t \rightarrow+\infty} \int_{-\infty}^{t} K_{1}(t-s) y_{1}(s) d s \leqslant \limsup _{t \rightarrow+\infty} y_{1}(t) \leqslant \frac{r_{1}-\frac{b_{1} m_{2}^{(1)}}{1+m_{2}^{(1)}}}{a_{1}} .
$$

Hence, for $\varepsilon>0$ which satisfies (3.1), it follows from (3.11)-(3.12) that there exists a $\mathrm{T}_{3}^{\prime}>0$ such that

$$
\begin{array}{r}
y_{1}(t)<\frac{r_{1}-\frac{b_{1} m_{2}^{(1)}}{1+m_{2}^{(1)}}}{a_{1}}+\frac{\varepsilon}{2} \stackrel{\text { def }}{=} M_{1}^{(2)} \text { for } t>T_{3}^{\prime}, \\
\int_{-\infty}^{t} K_{1}(t-s) y_{1}(s) d s<\frac{r_{1}-\frac{b_{1} m_{2}^{(1)}}{1+m_{2}^{(1)}}}{a_{1}}+\frac{\varepsilon}{2} \stackrel{\text { def }}{=} M_{1}^{(2)} \text { for } t>T_{3}^{\prime} .
\end{array}
$$


Similarly, for above $\varepsilon>0$, it follows from the second equation of system (1.1) that there exists a $T_{3}>T_{3}^{\prime}$ such that

$$
\begin{array}{r}
y_{2}(t)<\frac{r_{2}-\frac{b_{2} m_{1}^{(1)}}{1+m_{1}^{(1)}}}{a_{2}}+\frac{\varepsilon}{2} \stackrel{\text { def }}{=} M_{2}^{(2)} \text { for } t>T_{3}, \\
\int_{-\infty}^{t} K_{2}(t-s) y_{2}(s) d s<\frac{r_{2}-\frac{b_{2} m_{1}^{(1)}}{1+m_{1}^{(1)}}}{a_{2}}+\frac{\varepsilon}{2} \stackrel{\text { def }}{=} M_{2}^{(2)} \text { for } t>T_{3} .
\end{array}
$$

For $t>T_{3}$, it follows from the first equation of system (1.1), the strict increasing of function $f_{i}(x)=$ $\frac{b_{i} x}{1+\chi}, i=1,2$ and (3.14) that

$$
\frac{d y_{1}(t)}{d t} \geqslant y_{1}(t)\left[r_{1}-a_{1} y_{1}-\frac{b_{1} M_{2}^{(2)}}{1+M_{2}^{(2)}}\right] \text {. }
$$

Thus, as a direct corollary of Lemma 2.2, according to (3.15), one has

$$
\liminf _{t \rightarrow+\infty} y_{1}(t) \geqslant \frac{r_{1}-\frac{b_{1} M_{2}^{(2)}}{1+M_{2}^{(2)}}}{a_{1}}
$$

It follows from Lemma 2.3 that

$$
\liminf _{t \rightarrow+\infty} \int_{-\infty}^{t} K_{1}(t-s) y_{1}(s) d s \geqslant \limsup _{t \rightarrow+\infty} y_{1}(t) \geqslant \frac{r_{1}-\frac{b_{1} M_{2}^{(2)}}{1+M_{2}^{(2)}}}{a_{1}} .
$$

Hence, for $\varepsilon>0$ which satisfies (3.1), it follows from (3.16) and (3.17) that there exists a $\mathrm{T}_{4}^{\prime}>0$ such that

$$
\begin{aligned}
y_{1}(t)> & \frac{r_{1}-\frac{b_{1} M_{2}^{(2)}}{1+M_{2}^{(2)}}}{a_{1}}-\frac{\varepsilon}{2} \stackrel{\text { def }}{=} m_{1}^{(2)} \text { for } t>T_{4}^{\prime}, \\
\int_{-\infty}^{t} K_{1}(t-s) y_{1}(s) d s & >\frac{r_{1}-\frac{b_{1} M_{2}^{(2)}}{1+M_{2}^{(2)}}}{a_{1}}-\frac{\varepsilon}{2} \stackrel{\text { def }}{=} m_{1}^{(2)} \text { for } t>T_{4}^{\prime} .
\end{aligned}
$$

Similarly, for above $\varepsilon>0$, it follows from the second equation of system (1.1) that there exists a $T_{4}>T_{4}^{\prime}$ such that

$$
\begin{aligned}
y_{2}(t)> & \frac{r_{2}-\frac{b_{2} M_{1}^{(2)}}{1+M_{1}^{(2)}}}{a_{2}}-\frac{\varepsilon}{2} \stackrel{\text { def }}{=} m_{2}^{(2)} \text { for } t>T_{4}, \\
\int_{-\infty}^{t} K_{2}(t-s) y_{2}(s) d s & >\frac{r_{2}-\frac{b_{2} M_{1}^{(2)}}{1+M_{1}^{(2)}}}{a_{2}}-\frac{\varepsilon}{2} \stackrel{\text { def }}{=} m_{2}^{(2)} \text { for } t>T_{4} .
\end{aligned}
$$


One could easily see that

$$
\begin{aligned}
& M_{1}^{(2)}=\frac{r_{1}-\frac{b_{1} m_{2}^{(1)}}{1+m_{2}^{(1)}}}{a_{1}}+\frac{\varepsilon}{2}<\frac{r_{1}}{a_{1}}+\varepsilon=M_{1}^{(1)}, \\
& M_{2}^{(2)}=\frac{r_{2}-\frac{b_{2} m_{1}^{(1)}}{1+m_{1}^{(1)}}}{a_{2}}+\frac{\varepsilon}{2}<\frac{r_{2}}{a_{2}}+\varepsilon=M_{2}^{(1)}, \\
& m_{1}^{(2)}=\frac{r_{1}-\frac{b_{1} M_{2}^{(2)}}{1+M_{2}^{(2)}}}{a_{1}}-\frac{\varepsilon}{2}>\frac{r_{1}-\frac{b_{1} M_{2}^{(1)}}{1+M_{2}^{(1)}}}{a_{1}}-\varepsilon=m_{1}^{(1)}, \\
& m_{2}^{(2)}=\frac{r_{2}-\frac{b_{2} M_{1}^{(2)}}{1+M_{1}^{(2)}}-\frac{\varepsilon}{2}>\frac{r_{2}-\frac{b_{2} M_{1}^{(1)}}{1+M_{1}^{(1)}}}{a_{2}}-\varepsilon=m_{2}^{(1)} .}{a_{2}},
\end{aligned}
$$

Repeating the above procedure, we get four sequences $M_{i}^{(n)}, m_{i}^{(n)}, i=1,2, n=1,2, \ldots$, such that for $n \geqslant 2$

$$
\begin{aligned}
& M_{1}^{(n)}= \frac{r_{1}-\frac{b_{1} m_{2}^{(n-1)}}{1+m_{2}^{(n-1)}}}{a_{1}}+\frac{\varepsilon}{n}, \quad M_{2}^{(n)}=\frac{r_{2}-\frac{b_{2} m_{1}^{(n-1)}}{1+m_{1}^{(n-1)}}}{a_{2}}+\frac{\varepsilon}{n}, \\
& m_{1}^{(n)}=\frac{r_{1}-\frac{b_{1} M_{2}^{(n)}}{1+M_{2}^{(n)}}}{a_{1}}-\frac{\varepsilon}{n}, \quad m_{2}^{(n)}=\frac{r_{2}-\frac{b_{2} M_{1}^{(n)}}{1+M_{1}^{(n)}}-\frac{\varepsilon}{n} .}{a_{2}} .
\end{aligned}
$$

Obviously,

$$
m_{i}^{(n)}<N_{i}(t)<M_{i}^{(n)}, \text { for } t \geqslant T_{2 n}, i=1,2 .
$$

By induction, similarly to the analysis of Chen [2], we could prove that sequences $M_{i}^{(n)}, i=1,2$ are non-increasing, and sequences $m_{i}^{(n)}, i=1,2$ are non-decreasing. Therefore,

$$
\lim _{t \rightarrow+\infty} M_{i}^{(n)}=\bar{y}_{i}, \lim _{t \rightarrow+\infty} m_{i}^{(n)}=\underline{y}_{i}, i=1,2 .
$$

Letting $n \rightarrow+\infty$ in (3.13), we obtain

$$
a_{1} \bar{y}_{1}=r_{1}-\frac{b_{1} \underline{y}_{2}}{1+\underline{y}_{2}}, \quad a_{2} \underline{y}_{2}=r_{2}-\frac{b_{2} \bar{y}_{1}}{1+\bar{y}_{1}}, \quad a_{1} \underline{y}_{1}=r_{1}-\frac{b_{1} \bar{y}_{2}}{1+\bar{y}_{2}}, \quad a_{2} \bar{y}_{2}=r_{2}-\frac{b_{2} \underline{y}_{1}}{1+\underline{y}_{1}},
$$

(3.16) shows that $\left(\bar{y}_{1}, \underline{y}_{2}\right)$ and $\left(\underline{y}_{1}, \bar{y}_{2}\right)$ are solutions of the equation

$$
r_{1}-a_{1} y_{1}-\frac{b_{1} y_{2}}{1+y_{2}}=0, \quad r_{2}-a_{2} y_{2}-\frac{b_{2} y_{1}}{1+y_{1}}=0 .
$$

By Lemma 2.1, (3.18) has a unique positive solution $E^{*}\left(y_{1}^{*}, y_{2}^{*}\right)$. Hence, we conclude that

$$
\bar{y}_{i}=\underline{y}_{i}=y_{i}^{*}, \quad i=1,2 \text {, }
$$

that is

$$
\lim _{t \rightarrow+\infty} y_{i}(t)=y_{i}^{*} i=1,2 \text {. }
$$

Thus, the unique interior equilibrium $E^{*}\left(y_{1}^{*}, y_{2}^{*}\right)$ is globally attractive. This completes the proof of Theorem 1.1. 
Proof of Theorem 1.4. Condition

$$
r_{1}\left(a_{2}+r_{2}\right)>b_{1} r_{2}, r_{2}-\frac{b_{2} m_{1}}{1+m_{1}}<0
$$

implies that there exists an enough small $\varepsilon$ such that

$$
r_{1}>\frac{b_{1}\left(\frac{r_{2}}{a_{2}}+\varepsilon\right)}{1+\left(\frac{r_{2}}{a_{2}}+\varepsilon\right)}+a_{1} \varepsilon, \quad r_{2}-\frac{b_{2} m_{1}^{\varepsilon}}{1+m_{1}^{\varepsilon}}<0
$$

holds, where

$$
m_{1}^{\varepsilon}=\frac{r_{1}-\frac{b_{1} M_{2}^{\varepsilon}}{1+M_{2}^{\varepsilon}}}{a_{1}}, \quad M_{2}^{\varepsilon}=\frac{r_{2}}{a_{2}}+\varepsilon .
$$

Similarly to the analysis of (3.2)-(3.6), there exists a $T_{1}>0$ such that

$$
\begin{aligned}
y_{i}(t) & <\frac{r_{i}}{a_{i}}+\varepsilon=M_{i}^{\varepsilon}, \quad i=1,2, \\
\int_{-\infty}^{t} K_{i}(t-s) y_{i}(s) d s & <\frac{r_{i}}{a_{i}}+\varepsilon \stackrel{\text { def }}{=} M_{i}^{\varepsilon}, \quad i=1,2 .
\end{aligned}
$$

Now, for $t>T_{1}$, it follows from the first equation of system (1.1) and (3.21) that

$$
\frac{d y_{1}(t)}{d t} \geqslant y_{1}(t)\left[r_{1}-a_{1} y_{1}-\frac{b_{1} M_{2}^{\varepsilon}}{1+M_{2}^{\varepsilon}}\right]
$$

Thus, as a direct corollary of Lemma 2.2, according to (3.22), one has

$$
\liminf _{t \rightarrow+\infty} y_{1}(t) \geqslant \frac{r_{1}-\frac{b_{1} M_{2}^{\varepsilon}}{1+M_{2}^{\varepsilon}}}{a_{1}}
$$

It follows from Lemma 2.3 and (3.23) that

$$
\liminf _{t \rightarrow+\infty} \int_{-\infty}^{t} K_{1}(t-s) y_{1}(s) d s \geqslant \frac{r_{1}-\frac{b_{1} M_{2}^{\varepsilon}}{1+M_{2}^{\varepsilon}}}{a_{1}} .
$$

Hence, for enough small $\varepsilon>0$, which satisfies (3.19), it follows from (3.24) that there exists a $T_{2}>T_{1}$ such that for all $t>T_{2}$,

$$
\int_{-\infty}^{t} K_{1}(t-s) y_{1}(s) d s>\frac{r_{1}-\frac{b_{1} M_{2}^{\varepsilon}}{1+M_{2}^{\varepsilon}}}{a_{1}}-\varepsilon \stackrel{\text { def }}{=} m_{1}^{\varepsilon} \text { for } t>T_{2} .
$$

From the second equation of system (1.1) and (3.25), for $t>T_{2}$, we have

$$
\frac{d y_{2}(t)}{d t}<y_{2}(t)\left[r_{2}-\frac{b_{2} m_{1}^{\varepsilon}}{1+m_{1}^{\varepsilon}}\right]
$$

Hence,

$$
y_{2}(t)=y_{2}\left(T_{2}\right) \exp \left\{\int_{T_{2}}^{t}\left[r_{2}-\frac{b_{2} m_{1}^{\varepsilon}}{1+m_{1}^{\varepsilon}}\right] d t\right\} \rightarrow 0 \text { as } t \rightarrow+\infty .
$$


Equation (3.26) means that

$$
0 \leqslant \liminf _{t \rightarrow+\infty} y_{2}(t) \leqslant \limsup _{t \rightarrow+\infty} y_{2}(t) \leqslant 0
$$

Hence, by applying Lemma 2.3, we have

$$
\limsup _{t \rightarrow+\infty} \int_{-\infty}^{t} K_{2}(t-s) y_{2}(s) d s \leqslant \limsup _{t \rightarrow+\infty} y_{2}(t) \leqslant 0 .
$$

For $\varepsilon_{1}>0$ enough small, it follows from (3.27) that there exists a $T_{3}>T_{2}$ such that

$$
0<\int_{-\infty}^{t} K_{2}(t-s) y_{2}(s) d s<\varepsilon_{1} \text { for all } t \geqslant T_{3}
$$

It follows from (3.28), the strict increasing of function $f_{1}(x)=\frac{b_{1} x}{1+x}$, and the first equation of (1.1) that

$$
\frac{d y_{1}(t)}{d t}>y_{1}(t)\left[r_{1}-a_{1} y_{1}-\frac{b_{1} \varepsilon_{1}}{1+\varepsilon_{1}}\right]
$$

Applying Lemma 2.2 to (3.29), it follows that

$$
\lim _{t \rightarrow+\infty} y_{1}(t) \geqslant \frac{r_{1}-\frac{b_{1} \varepsilon_{1}}{1+\varepsilon_{1}}}{a_{1}} .
$$

Setting $\varepsilon \rightarrow 0$ leads to

$$
\lim _{t \rightarrow+\infty} y_{1}(t) \geqslant \frac{r_{1}}{a_{1}}
$$

This together with (3.20) shows that

$$
\lim _{t \rightarrow+\infty} y_{1}(t)=\frac{r_{1}}{a_{1}}
$$

This ends the proof of Theorem 1.1.

Proof of Theorem 1.5. Since the proof of Theorem 1.5 is similar to that of the proof of Theorem 1.4, we omit the detail here.

\section{Discussion}

In this paper, we propose a two-species competitive system with nonlinear inter-inhibition terms and infinite delay, which can be seen as the generalization of the model (1.3), by means of considering the past state of the species. By using the iterative method, we are able to obtain a set of sufficient conditions which ensure the global stability of the system. We also investigate the extinction property of the system (1.1). Our study indicates that the delay is both profitless and harmless.

\section{Acknowledgment}

The research was supported by the Natural Science Foundation of Fujian Province (2019J01783, 2019J01841). 


\section{References}

[1] F. D. Chen, On a nonlinear nonautonomous predator-prey model with diffusion and distributed delay, J. Comput. Appl. Math., 180 (2005), 33-49. 1, 1

[2] B. G. Chen, Global attractivity of a two-species competitive system with nonlinear inter-inhibition terms, J. Math. Computer Sci., 16 (2016), 481-494. 1, 1.2, 1.2, 1.6, 2, 3

[3] F. D. Chen, X. X. Chen, S. Y. Huang, Extinction of a two species non-autonomous competitive system with BeddingtonDeAngelis functional response and the effect of toxic substances, Open Math., 14 (2016), 1157-1173. 1, 1

[4] L. J. Chen, J. T. Sun, F. D. Chen, L. Zhao, Extinction in a Lotka-Volterra competitive system with impulse and the effect of toxic substances, Appl. Math. Model., 40 (2016), 2015-2024.

[5] F. D. Chen, H. N. Wang, Dynamic behaviors of a Lotka-Volterra competitive system with infinite delay and single feedback control, J. Nonlinear Funct. Anal., 2016 (2016), 11 pages. 1

[6] F. D. Chen, H. N. Wang, Y. H. Lin, W. L. Chen, Global stability of a stage-structured predator-prey system, Appl. Math. Comput., 223 (2013), 45-53. 1

[7] J. H. Chen, X. D. Xie, Stability analysis of a discrete competitive system with nonlinear interinhibition terms, Adv. Difference Equ., 2017 (2017), 19 pages.

[8] F. D. Chen, X. D. Xie, Z. S. Miao, L. Q. Pu, Extinction in two species nonautonomous nonlinear competitive system, Appl. Math. Comput., 274 (2016), 119-124. 1

[9] F. D. Chen, X. D. Xie, H. N. Wang, Global stability in a competition model of plankton allelopathy with infinite delay, J. Syst. Sci. Complex., 28 (2015), 1070-1079. 1

[10] F. M. de Oca, M. Vivas, Extinction in a two dimensional Lotka-Volterra system with infinite delay, Nonlinear Anal. Real World Appl., 7 (2006), 1042-1047. 2

[11] K. Gopalsamy, Stability and Oscillations in Delay Differential Equations of Population Dynamics, Kluwer Academic Publishers, Dordrecht, (1992). 1

[12] J. Hale, Theory of Functional Differential Equations, Springer-Verlag, New York-Heidelberg, (1977). 1

[13] R. Y. Han, X. D. Xie, F. D. Chen, Permanence and global attractivity of a discrete pollination mutualism in plant-pollinator system with feedback controls, Adv. Difference Equ., 2016 (2016), 17 pages.

[14] M. X. He, Z. Li, F. D. Chen, Dynamic of a nonautonomous two-species impulsive competitive system with infinite delays, Open Math., 17 (2019), 776-794.

[15] X. Y. Huang, F. D. Chen, X. D. Xie, L. Zhao, Extinction of a two species competitive stage-structured system with the effect of toxic substance and harvesting, Open Math., 17 (2019), 856-873.

[16] Z. Li, F. D. Chen, M. X. He, Asymptotic behavior of the reaction-diffusion model of plankton allelopathy with nonlocal delays, Nonlinear Anal. Real World Appl., 12 (2011), 1748-1758.

[17] Z. Li, F. D. Chen, M. X. He, Global stability of a delay differential equations model of plankton allelopathy, Appl. Math. Comput., 218 (2012), 7155-7163.

[18] T. X. Li, N. Pintus, G. Viglialoro, Properties of solutions to porous medium problems with different sources and boundary conditions, Z. Angew. Math. Phys., 70 (2019), 18 pages.

[19] Y. H. Lin, X. D. Xie, F. D. Chen, T. T. Li, Convergences of a stage-structured predator-prey model with modified LeslieGower and Holling-type II schemes, Adv. Difference Equ., 2016 (2016), 19 pages.

[20] Z. E. Ma, Mathematical Modeling and Study of Population Ecology (in Chinese), Hefei Anhui Education Publishing House, China, (1996). 1

[21] L. Q. Pu, X. D. Xie, F. D. Chen, Z. S. Miao, Extinction in two-species nonlinear discrete competitive system, Discrete Dyn. Nat. Soc., 2016 (2016), 10 pages.

[22] W. J. Qin, Z. J. Liu, Y. P. Chen, Permanence and global stability of positive periodic solutions of a discrete competitive system, Discrete Dyn. Nat. Soc., 2009 (2009), 13 pages.

[23] P. G. Wang, C. R. Li, J. Zhang, T. X. Li, Quasilinearization method for first-order impulsive integro-differential equations, Electron. J. Differential Equations, 2019 (2019), 14 pages.

[24] Q. L. Wang, Z. J. Liu, Z. X. Li, R. A. Cheke, Existence and global asymptotic stability of positive almost periodic solutions of a two-species competitive system, Int. J. Biomath., 7 (2014), 18 pages. 1

[25] R. X. Wu, L. Li, Q. F. Lin, A Holling type commensal symbiosis model involving Allee effect, Comm. Math. Biol. Neur., 2018 (2018), 13 pages.

[26] X. D. Xie, F. D. Chen, Y. L. Xue, Note on the stability property of a cooperative system incorporating harvesting, Discrete Dyn. Nat. Soc., 2014 (2014), 5 pages.

[27] X. D. Xie, F. D. Chen, K. Yang, Y. L. Xue, Global attractivity of an integro-differential model of mutualism, Abstract Appl. Anal., 2014 (2014), 6 pages.

[28] Y. L. Xue, X. D. Xie, Q. F. Lin Q, F. D. Chen, Global attractivity and extinction of a discrete competitive system with infinite delays and single feedback control, Discrete Dyn. Nat. Soc., 2018 (2018), 14 pages.

[29] K. Yang, Z. S. Miao, F. D. Chen, X. D. Xie, Influence of single feedback control variable on an autonomous Holling II type cooperative system, J. Math. Anal. Appl., 435 (2016), 874-888.

[30] K. Yang, X. D. Xie, F. D. Chen, Global stability of a discrete mutualism model, Abstr. Appl. Anal., 2014 (2014), 7 pages.

[31] S. B. Yu, Permanence for a discrete competitive system with feedback controls, Commun. Math. Biol. Neurosci., 2015 (2015), 11 pages. 
[32] S. B. Yu, F. D. Chen, Dynamic behaviors of a competitive system with Beddington-DeAngelis functional response, Discrete Dyn. Nat. Soc., 2019 (2019), 12 pages.

[33] Q. Yue, Dynamics of a modified Leslie-Gower predator-prey model with Holling-type II schemes and a prey refuge, SpringerPlus, 5 (2016), 12 pages.

[34] Q. Yue, Stability property of the prey free equilibrium point, Open Math., 17 (2019), 646-652. 1 